\title{
Some new Hardy-type inequalities for Riemann-Liouville fractional $q$-integral operator
}

\author{
Lars-Erik Persson ${ }^{1,2^{*}}$ and Serikbol Shaimardan ${ }^{3}$
}

"Correspondence: larserik@ltu.se 'Luleå University of Technology, Luleå, 97187 , Sweden

${ }^{2}$ Narvik University College, P.O. Box 385, Narvik, 8505, Norway Full list of author information is available at the end of the article

\begin{abstract}
We consider the $q$-analog of the Riemann-Liouville fractional $q$-integral operator of order $n \in N$. Some new Hardy-type inequalities for this operator are proved and discussed.

MSC: Primary 26D10; 26D15; secondary 33D05; 39A13

Keywords: inequalities; Hardy-type inequalities; Riemann-Liouville operator; integral operator; q-calculus; q-integral
\end{abstract}

\section{Introduction}

In $1910 \mathrm{FH}$ Jackson defined $q$-derivative and definite $q$-integral [1] (see also [2]). It was the starting point of $q$-analysis. Today the interest in the subject has exploded. The $q$-analysis has numerous applications in various fields of mathematics, e.g., dynamical systems, number theory, combinatorics, special functions, fractals and also for scientific problems in some applied areas such as computer science, quantum mechanics and quantum physics (see, e.g., [3-7]). For further development and recent results in $q$-analysis, we refer to the books $[2,3]$ and [5] and the references given therein. The first results concerning integral inequalities in $q$-analysis were proved in 2004 by Gauchman [8]. Later on some further $q$-analogs of the classical inequalities have been proved (see [9-12]). Moreover, in 2014 Maligranda et al. [13] derived a $q$-analog of the classical Hardy inequality. Further development of Hardy's original inequality from 1925 (see [14] and [15]) has been enormous. Some of the most important results and applications have been presented and discussed in the books $[16,17]$ and [18]. Hence, it seems to be a huge new research area to investigate which of these so-called Hardy-type inequalities have their $q$-analogs.

The aim of this paper is to obtain some $q$-analogs of Hardy-type inequalities for the Riemann-Liouville fractional integral operator of order $n \in \mathbf{N}$ and to find necessary and sufficient conditions of the validity of these inequalities for all non-negative real functions (see Theorems 3.1 and 3.2).

The paper is organized as follows. In order not to disturb our discussions later on, some preliminaries are presented in Section 2. The main results can be found in Section 3, while the detailed proofs are given in Section 4.

(c) 2015 Persson and Shaimardan. This article is distributed under the terms of the Creative Commons Attribution 4.0 International License (http://creativecommons.org/licenses/by/4.0/), which permits unrestricted use, distribution, and reproduction in any medium, provided you give appropriate credit to the original author(s) and the source, provide a link to the Creative Commons license, and indicate if changes were made. 


\section{Preliminaries}

First we recall some definitions and notations in $q$-analysis from the recent books $[2,3]$ and [5].

Let $q \in(0,1)$. Then a $q$-real number $[\alpha]_{q}$ is defined by

$$
[\alpha]_{q}:=\frac{1-q^{\alpha}}{1-q}, \quad \alpha \in \mathrm{R}
$$

where $\lim _{q \rightarrow 1} \frac{1-q^{\alpha}}{1-q}=\alpha$.

The $q$-analog of the binomial coefficients is defined by

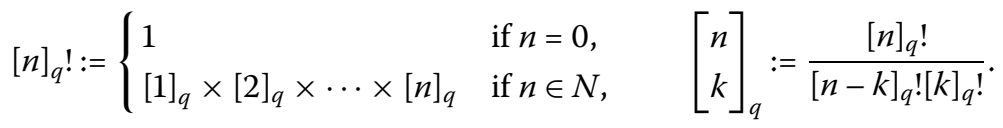

We introduce the $q$-analog of a polynomial in the following way:

$$
\begin{aligned}
& (x-a)_{q}^{n}:= \begin{cases}1 & \text { if } n=0, \\
(x-a)(x-q a) \cdots\left(x-q^{n-1} a\right) & \text { if } n \in N,\end{cases} \\
& (x-a)_{q}^{n+m}=(x-a)_{q}^{m}\left(x-q^{m} a\right)_{q}^{n}, \quad n, m=0,1,2, \ldots .
\end{aligned}
$$

The $q$-gamma function $\Gamma_{q}$ is defined by

$$
\Gamma_{q}(n+1):=[n]_{q} !, \quad n \in N .
$$

For $f:[0, b) \rightarrow R, 0<b \leq \infty$, we define the $q$-derivative as follows:

$$
D_{q} f(x):=\frac{f(x)-f(q x)}{(1-q) x}, \quad x \in[0, b)
$$

Clearly, if the function $f(x)$ is differentiable at a point $x \in(0,1)$, then $\lim _{q \rightarrow 1} D_{q} f(x)=f^{\prime}(x)$. Let $0<a \leq b<\infty$. The definite $q$-integral (also called the $q$-Jackson integral) of a function $f(x)$ is defined by the formulas

$$
\int_{0}^{a} f(x) d_{q} x:=(1-q) a \sum_{k=0}^{\infty} q^{k} f\left(q^{k} a\right)
$$

Moreover, the improper $q$-integral of a function $f(x)$ is defined by

$$
\int_{0}^{\infty} f(x) d_{q} x:=(1-q) \sum_{k=-\infty}^{\infty} q^{k} f\left(q^{k}\right)
$$

provided that the series on the right-hand sides of (3) and (4) converge absolutely.

Suppose that $f(x)$ and $g(x)$ are two functions which are defined on $(0, \infty)$. Then

$$
\int_{0}^{\infty} f(x) D_{q}(g(x))=\sum_{j=0}^{\infty} f\left(q^{j}\right)\left(g\left(q^{j}\right)-g\left(q^{j+1}\right)\right) .
$$


Let $\Omega$ be a subset of $(0, \infty)$ and $\mathcal{X}_{\Omega}(t)$ denote the characteristic function of $\Omega$. For all $z$ : $0<z<\infty$, we have that

$$
\int_{0}^{\infty} \mathcal{X}_{(0, z]}(t) f(t) d_{q} t=(1-q) \sum_{q^{i} \leq z} q^{i} f\left(q^{i}\right)
$$

and

$$
\int_{0}^{\infty} \mathcal{X}_{[z, \infty)}(t) f(t) d_{q} t=(1-q) \sum_{q^{i} \geq z} q^{i} f\left(q^{i}\right) .
$$

Al-Salam (see [19] and also [3]) introduced the fractional $q$-integral of the RiemannLiouville operator $\mathcal{I}_{q, n}$ of order $n \in \mathbf{N}$ by

$$
\mathcal{I}_{q, n} f(x):=\frac{1}{\Gamma_{q}(n)} \int_{0}^{x} K_{n-1}(x, s) f(s) d_{q} s,
$$

where $K_{n-1}(x, s)=(x-q s)_{q}^{n-1}$.

Next we will present a lemma (Lemma 2.1) concerning discrete Hardy-type inequalities which are proved in [20]. In this paper all authors studied inequalities of the form

$$
\left(\sum_{j=1}^{\infty} u_{j}^{r}\left(\left(S_{n} f\right)_{j}\right)^{r}\right)^{\frac{1}{r}} \leq C\left(\sum_{i=1}^{\infty} v_{i}^{p} f_{i}^{p}\right)^{\frac{1}{p}}, \quad \forall f \geq 0
$$

for the $n$-multiple discrete Hardy operator with weights of the form

$$
\left(S_{n} f\right)_{j}=\sum_{k_{1}=j}^{\infty} \omega_{1, k_{1}} \sum_{k_{2}=1}^{k_{1}} \omega_{2, k_{2}} \sum_{k_{3}=1}^{k_{2}} \omega_{3, k_{3}} \ldots \sum_{k_{n-1}=1}^{k_{n-2}} \omega_{n-1, k_{n-1}} \sum_{i=1}^{k_{n-1}} f_{i}=\sum_{i=j}^{\infty} A_{n-1}(i, j) f_{i},
$$

where $u=\left\{u_{i}\right\}_{i=1}^{\infty}, v=\left\{v_{i}\right\}_{i=1}^{\infty}, \omega_{i}=\left\{\omega_{i, k}\right\}_{k=1}^{\infty}$ are positive sequences of real numbers (i.e., weight sequences). She also studied inequality (8) for the operator $S_{n}^{*}$ defined by

$$
\left(S_{n}^{*} f\right)_{i}:=\sum_{j=1}^{i} f_{i} A_{n-1,1}(i, j)
$$

which is the conjugate to the operator $S_{n}$, where $A_{n-1,1}(i, j) \equiv 1$ for $n=1$ and

$$
A_{n-1,1}(i, j)=\sum_{k_{n-1}=j}^{i} \omega_{n-1, k_{n-1}} \sum_{k_{n-2}=k_{n-1}}^{i} \omega_{n-2, k_{n-2}} \cdots \sum_{k_{1}=k_{2}}^{i} \omega_{1, k_{1}}
$$

for $n \geq 2$.

We consider the following Hardy-type inequalities:

$$
\left(\sum_{j=-\infty}^{\infty} u_{j}^{r}\left(\left(S_{n} f\right)_{j}\right)^{r}\right)^{\frac{1}{r}} \leq C\left(\sum_{i=-\infty}^{\infty} v_{i}^{p} f_{i}^{p}\right)^{\frac{1}{p}}
$$

and

$$
\left(\sum_{i=-\infty}^{\infty} u_{i}^{r}\left(\left(S_{n}^{*} f\right)_{i}\right)^{r}\right)^{\frac{1}{r}} \leq C^{*}\left(\sum_{i=-\infty}^{\infty} v_{i}^{p} f_{i}^{p}\right)^{\frac{1}{p}} .
$$


In the sequel, for any $p>1$, the conjugate number $p^{\prime}$ is defined by $p^{\prime}:=p /(p-1)$, and the considered functions are assumed to be non-negative. Moreover, the symbol $M \ll K$ means that there exists $\alpha>0$ such that $M \leq \alpha K$, where $\alpha$ is a constant which may depend only on parameters such as $p, q$, $r$. Similarly, the case $K \ll M$. If $M \ll K \ll M$, then we write $M \approx K$.

\section{Lemma 2.1}

(i) Let $1<p \leq r<\infty$ and $n \geq 1$. Then inequality (9) holds if and only if $\mathfrak{A}(n)=\max _{0 \leq m \leq n-1} \mathfrak{A}_{m}(n)<\infty$, where

$$
\mathfrak{A}_{m}(n)=\sup _{k \in \mathbb{Z}}\left(\sum_{j=k}^{\infty} A_{m, 1}^{p^{\prime}}(j, k) v_{j}^{-p^{\prime}}\right)^{\frac{1}{p^{\prime}}}\left(\sum_{i=-\infty}^{k} A_{n-1, m+1}^{r}(k, i) u_{i}^{r}\right)^{\frac{1}{r}}, \quad n \in \mathbb{N} .
$$

Moreover, $\mathfrak{A}(n) \approx C$, where $C$ is the best constant in (9).

(ii) Let $1<p \leq r<\infty$ and $n \geq 1$. Then inequality (10) holds if and only if $\mathfrak{A}^{*}(n)=\max _{0 \leq m \leq n-1} \mathfrak{A}^{*}{ }_{m}(n)<\infty$, where

$$
\mathfrak{A}_{m}^{*}(n)=\sup _{k \in \mathbb{Z}}\left(\sum_{i=k}^{\infty} A_{m, 1}^{r}(i, k) u_{i}^{r}\right)^{\frac{1}{r}}\left(\sum_{j=-\infty}^{k} A_{n-1, m+1}^{p^{\prime}}(k, j) v_{j}^{-p^{\prime}}\right)^{\frac{1}{p^{\prime}}}, \quad n \in \mathbb{N} .
$$

Moreover, $\mathfrak{A}^{*}(n) \approx C$, where $C$ is the best constant in (10).

We also need the corresponding result for the case $1<r<p<\infty$, which was proved in [21].

\section{Lemma 2.2}

(i) Let $1<r<p<\infty$ and $n \geq 1$. Then inequality (9) holds if and only if $\mathfrak{B}(n)=\max _{0 \leq m \leq n-1} \mathfrak{B}_{m}(n)<\infty$, where

$$
\begin{aligned}
\mathfrak{B}_{m}(n)= & \left\{\sum_{i=-\infty}^{\infty}\left(\sum_{j=i}^{\infty} A_{m, 1}^{p^{\prime}}(j, i) v_{j}^{-p^{\prime}}\right)^{\frac{p(r-1)}{p-r}}\left(\sum_{k=-\infty}^{i} A_{n-1, m+1}^{r}(i, k) u_{k}^{r}\right)^{\frac{p}{p-r}}\right. \\
& \left.\times \Delta^{+}\left(\sum_{j=i}^{\infty} A_{m, 1}^{p^{\prime}}(j, i) v_{j}^{-p^{\prime}}\right)\right\}^{\frac{p-r}{p r}}, \quad \Delta^{+} E_{i, j}=E_{i, j}-E_{i, j+1}, n \in \mathbb{N} .
\end{aligned}
$$

Moreover, $\mathfrak{B}(n) \approx C$, where $C$ is the best constant in (9).

(ii) Let $1<r<p<\infty$ and $n \geq 1$. Then inequality (10) holds if and only if $\mathfrak{B}^{*}(n)=\max _{0 \leq m \leq n-1} \mathfrak{B}^{*}{ }_{m}(n)<\infty$, where

$$
\begin{aligned}
\mathfrak{B}_{m}^{*}(n)= & \left\{\sum_{i=-\infty}^{\infty}\left(\sum_{j=i}^{\infty} A_{m, 1}^{r}(j, i) u_{j}^{r}\right)^{\frac{r}{p-r}}\left(\sum_{k=-\infty}^{i} A_{n-1, m+1}^{p^{\prime}}(i, k) v_{k}^{-p^{\prime}}\right)^{\frac{r(p-1)}{p-r}}\right. \\
& \left.\times \triangle^{+}\left(\sum_{j=i}^{\infty} A_{m, 1}^{r}(j, i) u_{j}^{r}\right)\right\}^{\frac{p-r}{p r}}, \quad \triangle^{+} E_{i, j}=E_{i, j}-E_{i, j+1}, \forall n \in \mathbb{N} .
\end{aligned}
$$

Moreover, $\mathfrak{B}^{*}(n) \approx C^{*}$, where $C^{*}$ is the best constant in $(10)$. 
Let $\left(a_{i, j}^{(n)}\right)$ be a matrix whose elements are non-negative and non-increasing in the second index for all $i, j: \infty>i \geq j>-\infty$, and the entries of the matrix $a_{i, j}^{(n)}$ satisfy the following (socalled discrete Oinarov condition):

$$
a_{i, j}^{(n)} \approx \sum_{\gamma=0}^{n} a_{i, k}^{(\gamma)} d_{k, j}^{n, \gamma}, \quad \gamma=1,2, \ldots, n-1, n \in \mathbb{N}
$$

for all $\infty>i \geq k \geq j>-\infty$.

Remark 2.3 Note that the matrices $\left(d_{k, j}^{\gamma, m}\right), \gamma=0,1, \ldots, m, m \geq 0$, are arbitrary nonnegative matrices which satisfy (11) (see [22]).

Moreover, in [22] necessary and sufficient conditions for inequalities (9) and (10) were proved for matrix operators with a matrix $\left(a_{i, j}^{(n)}\right)$ which satisfies (11). For our purposes we need such characterization on the following form.

\section{Lemma 2.4}

(i) Let $1<p \leq r<\infty$ and the entries of the matrix $\left(a_{i, j}^{(n)}\right)$ satisfy condition (11). Then inequality (9) for the operator $\left(\mathcal{A}^{-} f\right)_{j}:=\sum_{i=j}^{\infty} a_{i, j}^{(n)} f_{i}, j \in Z$, holds if and only if at least one of the conditions $\mathfrak{B}^{+}<\infty$ or $\mathfrak{B}^{-}<\infty$ holds, where

$$
\begin{aligned}
& \mathfrak{B}^{-}=\sup _{k \in Z}\left(\sum_{i=k}^{\infty} v_{i}^{-p^{\prime}}\left(\sum_{j=-\infty}^{k}\left(a_{i, j}^{(n)}\right)^{r} u_{j}^{r}\right)^{\frac{p^{\prime}}{r}}\right)^{\frac{1}{p^{\prime}}}, \\
& \mathfrak{B}^{+}=\sup _{k \in Z}\left(\sum_{j=-\infty}^{k} u_{j}^{r}\left(\sum_{i=k}^{\infty}\left(a_{i, j}^{(n)}\right)^{p^{\prime}} v_{i}^{-p^{\prime}}\right)^{\frac{r}{p^{\prime}}}\right)^{\frac{1}{r}} .
\end{aligned}
$$

Moreover, $\mathfrak{B}^{+} \approx \mathfrak{B}^{-} \approx C$, where $C$ is the best constant in (9).

(ii) Let $1<p \leq r<\infty$. Let the entries of the matrix $\left(a_{i, j}^{(n)}\right)$ satisfy condition (11). Then inequality (10) for the operator $\left(\mathcal{A}^{+} f\right)_{i}:=\sum_{j=-\infty}^{i} a_{i, j}^{(n)} f_{j}, i \in Z$, holds if and only if at least one of the conditions $\mathfrak{A}^{+}<\infty$ or $\mathfrak{A}^{-}<\infty$ holds, where

$$
\begin{aligned}
& \mathfrak{A}^{-}=\sup _{k \in Z}\left(\sum_{i=k}^{\infty} u_{i}^{r}\left(\sum_{j=-\infty}^{k}\left(a_{i, j}^{(n)}\right)^{p^{\prime}} v_{j}^{-p^{\prime}}\right)^{\frac{r}{p^{\prime}}}\right)^{\frac{1}{r}}, \\
& \mathfrak{A}^{+}=\sup _{k \in Z}\left(\sum_{j=-\infty}^{k} v_{j}^{-p^{\prime}}\left(\sum_{i=k}^{\infty}\left(a_{i, j}^{(n)}\right)^{r} u_{i}^{r}\right)^{\frac{p^{\prime}}{r}}\right)^{\frac{1}{p^{\prime}}} .
\end{aligned}
$$

Moreover, $\mathfrak{A}^{+} \approx \mathfrak{A}^{-} \approx C$, where $C$ is the best constant in (10).

\section{The main results}

Let $1<r, p \leq \infty$. Then the $q$-analog of the two-weighted inequality for the operator $\mathcal{I}_{q, n}$ of the form

$$
\left(\int_{0}^{\infty} u^{r}(x)\left(\mathcal{I}_{q, n} f(x)\right)^{r} d_{q} x\right)^{\frac{1}{r}} \leq C\left(\int_{0}^{\infty} \nu^{p}(x) f^{p}(x) d_{q} x\right)^{\frac{1}{p}}
$$


has several applications in various fields of science. In the classical analysis two-weighted estimates for the Riemann-Liouville fractional operator were derived by Stepanov for the case with parameters greater than one (see [23, 24]).

We consider the operator $I_{q, n}$ of the following form:

$$
I_{q, n} f(x)=\frac{1}{\Gamma_{q}(n)} \int_{0}^{\infty} \mathcal{X}_{(0, x]}(s) K_{n-1}(x, s) f(s) d_{q} s
$$

which is defined for all $x>0$. Although it does not coincide with the operator $\mathcal{I}_{q, n}$ (they coincide at the points $x=q^{k}, k \in \mathbb{Z}$ ), we have the equality

$$
\int_{0}^{\infty} u^{r}(x)\left(\mathcal{I}_{q, n} f(x)\right)^{r} d_{q} x=\int_{0}^{\infty} u^{r}(x)\left(I_{q, n} f(x)\right)^{r} d_{q} x
$$

Therefore, inequality (12) can be rewritten as

$$
\left(\int_{0}^{\infty} u^{r}(x)\left(I_{q, n} f(x)\right)^{r} d_{q} x\right)^{\frac{1}{r}} \leq C\left(\int_{0}^{\infty} v^{p}(x) f^{p}(x) d_{q} x\right)^{\frac{1}{p}}
$$

Its conjugate operator $I_{q, n}^{*}$ can be defined by

$$
I_{q, n}^{*} f(s):=\frac{1}{\Gamma_{q}(n)} \int_{0}^{\infty} \mathcal{X}_{[s, \infty)}(x) K_{n-1}(x, s) f(x) d_{q} x,
$$

with the same kernel. The dual inequality of inequality (13) reads as follows:

$$
\left(\int_{0}^{\infty} u^{r}(x)\left(I_{q, n}^{*} f(x)\right)^{r} d_{q} x\right)^{\frac{1}{r}} \leq C^{*}\left(\int_{0}^{\infty} \nu^{p}(x) f^{p}(x) d_{q} x\right)^{\frac{1}{p}}
$$

where $C$ and $C^{*}$ are positive constants independent of $f$ and $u(\cdot), v(\cdot)$ are positive realvalued functions on $(0, \infty)$, i.e., weight functions. In what follows we investigate inequalities (13) and (14).

Let $\mathbf{N}_{\mathbf{0}}=\mathbf{N} \cup\{0\}$. Then, for $0 \leq m \leq n-1, m, n \in \mathbf{N}_{\mathbf{0}}$, we use the following notations:

$$
\begin{aligned}
Q_{m}^{n-1}= & \left\{\int_{0}^{\infty}\left(\int_{0}^{\infty} \mathcal{X}_{(0, z]}(s) K_{m}^{p^{\prime}}(z, s) v^{-p^{\prime}}(s) d_{q} s\right)^{\frac{p(r-1)}{p-r}}\right. \\
& \times\left(\int_{0}^{\infty} \mathcal{X}_{[z, \infty)}(x) K_{n-m-1}^{r}(x, z) u^{r}(x) d_{q} x\right)^{\frac{p}{p-r}} \\
& \left.\times D_{q}\left(\int_{0}^{\infty} \mathcal{X}_{(0, z]}(s) K_{m}^{p^{\prime}}(z, s) v^{-p^{\prime}}(s) d_{q} s\right)\right\}^{\frac{p-r}{p r}}, \\
\mathcal{Q}_{m}^{n-1}= & \left\{\int_{0}^{\infty}\left(\int_{0}^{\infty} \mathcal{X}_{(0, z]}(s) K_{m}^{r}(z, s) u^{r}(s) d_{q} s\right)^{\frac{r}{p-r}}\right. \\
& \times\left(\int_{0}^{\infty} \mathcal{X}_{[z, \infty)}(x) K_{n-m-1}^{-p^{\prime}}(z, x) v^{-p^{\prime}}(x) d_{q} x\right)^{\frac{r(p-1)}{p-r}} \\
& \left.\times D_{q}\left(\int_{0}^{\infty} \mathcal{X}_{(0, z]}(s) K_{m}^{r}(z, s) u^{r}(x) d_{q} s\right)\right\}^{\frac{p-r}{p r}},
\end{aligned}
$$




$$
\begin{aligned}
& H_{m}^{n-1}=\sup _{z>0}\left(\int_{0}^{\infty} \mathcal{X}_{[z, \infty)}(x) K_{n-m-1}^{r}(x, z) u^{r}(x) d_{q} x\right)^{\frac{1}{r}}\left(\int_{0}^{\infty} \mathcal{X}_{(0, z]}(s) K_{m}^{p^{\prime}}(z, s) v^{-p^{\prime}}(s) d_{q} s\right)^{\frac{1}{p^{\prime}}}, \\
& \mathcal{H}_{m}^{n-1}=\sup _{z>0}\left(\int_{0}^{\infty} \mathcal{X}_{(0, z]}(x) K_{m}^{r}(z, x) u^{r}(x) d_{q} x\right)^{\frac{1}{r}}\left(\int_{0}^{\infty} \mathcal{X}_{[z, \infty)}(s) K_{n-m-1}^{p^{\prime}}(s, z) v^{-p^{\prime}}(s) d_{q} s\right)^{\frac{1}{p^{\prime}}} \text {, } \\
& A^{+}(z)=\left(\int_{0}^{\infty} \mathcal{X}_{[z, \infty)}(x) u^{r}(x)\left(\int_{0}^{\infty} \mathcal{X}_{(0, z]}(t) K_{n-1}^{p^{\prime}}(x, t) v^{-p^{\prime}}(t) d_{q} t\right)^{\frac{r}{p^{\prime}}} d_{q} x\right)^{\frac{1}{r}}, \\
& A^{-}(z)=\left(\int_{0}^{\infty} \mathcal{X}_{(0, z]}(t) v^{-p^{\prime}}(t)\left(\int_{0}^{\infty} \mathcal{X}_{[z, \infty)}(x) K_{n-1}^{r}(x, t) u^{r}(x) d_{q} x\right)^{\frac{p^{\prime}}{r}} d_{q} t\right)^{\frac{1}{p^{\prime}}} . \\
& \mathcal{A}^{+}(z)=\left(\int_{0}^{\infty} \mathcal{X}_{[z, \infty)}(t) v^{-p^{\prime}}(t)\left(\int_{0}^{\infty} \mathcal{X}_{(0, z]}(x) K_{n-1}^{r}(t, x) u^{r}(x) d_{q} x\right)^{\frac{p^{\prime}}{r}} d_{q} t\right)^{\frac{1}{p^{\prime}}}, \\
& \mathcal{A}^{-}(z)=\left(\int_{0}^{\infty} \mathcal{X}_{(0, z]}(x) u^{r}(x)\left(\int_{0}^{\infty} \mathcal{X}_{[z, \infty)}(t) K_{n-1}^{p^{\prime}}(t, x) v^{-p^{\prime}}(t) d_{q} t\right)^{\frac{r}{p^{\prime}}} d_{q} x\right)^{\frac{1}{r}}, \\
& H_{n-1}=\max _{0 \leq k \leq n-1} H_{k}^{n-1}, \quad \mathcal{H}_{n-1}=\max _{0 \leq k \leq n-1} \mathcal{H}_{k}^{n-1}, \\
& A_{q}^{+}=\sup _{z>0} A^{+}(z), \quad A_{q}^{-}=\sup _{z>0} A^{-}(z), \quad \mathcal{A}_{q}^{+}=\sup _{z>0} \mathcal{A}^{+}(z), \quad \mathcal{A}_{q}^{-}=\sup _{z>0} \mathcal{A}^{-}(z), \\
& Q_{n-1}=\max _{0 \leq k \leq n-1} Q_{k}^{n-1} \text { and } \mathcal{Q}_{n-1}=\max _{0 \leq k \leq n-1} \mathcal{Q}_{k}^{n-1} \text {. }
\end{aligned}
$$

Our main results read as follows.

\section{Theorem 3.1}

(i) Let $1<r<p<\infty$. Then inequality (13) holds if and only if $Q_{n-1}<\infty$. Moreover, $Q_{n-1} \approx C$, where $C$ is the best constant in (13).

(ii) Let $1<p \leq r<\infty$. Then inequality (13) holds if and only if at least one of the conditions $H_{n-1}<\infty$ or $A_{q}^{+}<\infty$ or $A_{q}^{-}<\infty$ holds. Moreover, $H_{n-1} \approx A_{q}^{+} \approx A_{q}^{-} \approx C$, where $C$ is the best constant in (13).

\section{Theorem 3.2}

(i) Let $1<r<p<\infty$. Then inequality (14) holds if and only if $\mathcal{Q}_{n-1}<\infty$. Moreover, $\mathcal{Q}_{n-1} \approx C^{*}$, where $C^{*}$ is the best constant in (14).

(ii) Let $1<p \leq r<\infty$. Then inequality (14) holds if and only if at least one of the conditions $\mathcal{H}_{n-1}<\infty$ or $\mathcal{A}_{q}^{+}<\infty$, or $\mathcal{A}_{q}^{-}<\infty$ holds. Moreover, $\mathcal{H}_{n-1} \approx \mathcal{A}_{q}^{+} \approx \mathcal{A}_{q}^{-} \approx C$, where $C$ is the best constant in (14).

For the proofs of these results, we need the following lemmata of independent interest.

Lemma 3.3 Let $x, t, s: 0<s \leq t \leq x<\infty$. Then

$$
\max _{0 \leq m \leq n-1} K_{n-m-1}(x, t) K_{m}(t, s) \leq K_{n-1}(x, s) \leq \sum_{m=0}^{n-1}\left[\begin{array}{c}
n-1 \\
m
\end{array}\right]_{q} K_{n-m-1}(x, t) K_{m}(t, s)
$$

for $m: 0 \leq m \leq n-1, n, m-1 \in \mathbb{N}$ and where $K_{n-1}(x, s)=(x-q s)_{q}^{n-1}$.

Lemma 3.4 Let $f$ and $g$ be non-negative functions on $(0, \infty), \alpha, \beta \in \mathbb{R}$ and

$$
I(z):=\left(\int_{0}^{\infty} \mathcal{X}_{(0, z]}(t) f(t) d_{q} t\right)^{\alpha}\left(\int_{0}^{\infty} \mathcal{X}_{[z, \infty)}(x) g(x) d_{q} x\right)^{\beta} .
$$


Then

$$
\sup _{z>0} I(z)=(1-q)^{\alpha+\beta} \sup _{k \in \mathbb{Z}}\left(\sum_{j=k}^{\infty} q^{j} f\left(q^{j}\right)\right)^{\alpha}\left(\sum_{i=-\infty}^{k} q^{i} g\left(q^{i}\right)\right)^{\beta},
$$

where at least one of $\alpha$ and $\beta$ is non-negative.

This result was proved in [25], but for the readers' convenience we will include in Section 4 a proof which is slightly simpler than that in the Russian version given in [25].

Lemma 3.5 Let $\alpha, \beta \in \mathbb{R}^{+}, K(\cdot, \cdot)$ be a non-negative function and

$$
\begin{aligned}
& \mathcal{I}^{+}(z):=\left(\int_{0}^{\infty} \mathcal{X}_{[z, \infty)}(x) g(x)\left(\int_{0}^{\infty} \mathcal{X}_{(0, z]}(t) K(x, t) f(t) d_{q} t\right)^{\alpha} d_{q} x\right)^{\beta}, \\
& \mathcal{I}^{-}(z):=\left(\int_{0}^{\infty} \mathcal{X}_{(0, z]}(t) f(t)\left(\int_{0}^{\infty} \mathcal{X}_{[z, \infty)}(x) K(x, t) g(x) d_{q} x\right)^{\alpha} d_{q} t\right)^{\beta} .
\end{aligned}
$$

Then

$$
\sup _{z>0} \mathcal{I}^{+}(z)=\sup _{k \in \mathbb{Z}}\left((1-q) \sum_{j=-\infty}^{k} q^{j} g\left(q^{j}\right)\left((1-q) \sum_{i=k}^{\infty} q^{i} K\left(q^{j}, q^{i}\right) f\left(q^{i}\right)\right)^{\alpha}\right)^{\beta}
$$

and

$$
\sup _{z>0} \mathcal{I}^{-}(z)=\sup _{k \in \mathbb{Z}}\left((1-q) \sum_{j=k}^{\infty} q^{j} f\left(q^{j}\right)\left((1-q) \sum_{j=-\infty}^{k} q^{j} K\left(q^{j}, q^{i}\right) g\left(q^{j}\right)\right)^{\alpha}\right)^{\beta} .
$$

Lemma 3.6 Let $Q_{m}^{n-1}, \mathcal{Q}_{m}^{n-1}<\infty$ for $0<m \leq n-1$. Then

$$
\begin{aligned}
Q_{m}^{n-1}= & \left\{\sum_{i=-\infty}^{\infty}\left((1-q) \sum_{t=i}^{\infty} q^{t} K_{m}^{p^{\prime}}\left(q^{i}, q^{t}\right) v^{-p^{\prime}}\left(q^{t}\right)\right)^{\frac{p(r-1)}{p-r}}\right. \\
& \times\left((1-q) \sum_{j=-\infty}^{i} q^{j} K_{n-m-1}^{r}\left(q^{j}, q^{i}\right) u^{r}\left(q^{j}\right)\right)^{\frac{p}{p-r}} \\
& \left.\times \triangle^{+}\left(\sum_{n=i}^{\infty}(1-q) q^{n} K_{m}^{r}\left(q^{i}, q^{n}\right) v^{-p^{\prime}}\left(q^{n}\right)\right)\right\}^{\frac{p-r}{p r}}
\end{aligned}
$$

and

$$
\begin{aligned}
\mathcal{Q}_{m}^{n-1}= & \left\{\sum_{i=-\infty}^{\infty}\left((1-q) \sum_{t=i}^{\infty} q^{t} K_{m}^{r}\left(q^{i}, q^{t}\right) u^{r}\left(q^{t}\right)\right)^{\frac{r}{p-r}}\right. \\
& \times\left((1-q) \sum_{j=-\infty}^{i} q^{j} K_{n-m-1}^{p^{\prime}}\left(q^{j}, q^{i}\right) v^{-p^{\prime}}\left(q^{j}\right)\right)^{\frac{r(p-1)}{p-r}} \\
& \left.\times \Delta^{+}\left(\sum_{n=i}^{\infty}(1-q) q^{n} K_{m}^{r}\left(q^{i}, q^{n}\right) u^{r}\left(q^{n}\right)\right)\right\}^{\frac{p-r}{p r}}
\end{aligned}
$$

where $\triangle^{+} E_{n, i}=E_{n, i}-E_{n, i+1}$. 


\section{Proofs}

Proof of Lemma 3.3 Let $0<s \leq t \leq x<\infty$. First we prove the lower estimate. By using (2) we find that

$$
\begin{aligned}
K_{n-m-1}(x, t) K_{m}(t, s) & =(x-q t)_{q}^{n-m-1}(t-q s)_{q}^{m} \\
& \leq(x-q s)_{q}^{n-m-1}(x-q s)_{q}^{m} \\
& \leq(x-q s)_{q}^{n-m-1}\left(x-q^{n-m} s\right)_{q}^{m} \\
& =(x-q s)_{q}^{n-1}=K_{n-1}(x, s)
\end{aligned}
$$

for $0<s \leq t \leq x<\infty$ and $0 \leq m \leq n-1, m-1, n \in \mathbb{N}$. Hence,

$$
\max _{0 \leq m \leq n-1} K_{n-m-1}(x, t) K_{m}(t, s) \leq K_{n-1}(x, s)
$$

and the lower estimate in (15) is proved.

According to (1) we get that $K_{0}(x, t) K_{0}(t, s)=K_{0}(x, s) \equiv 1$ for $n=1$. Moreover, we have that

$$
\begin{aligned}
K_{1}(x, s) & =(x-q s)_{q}<(x-q t)_{q}+(t-q s)_{q} \\
& =\sum_{m=0}^{1}\left[\begin{array}{c}
1 \\
m
\end{array}\right]_{q} K_{2-m-1}(x, t) K_{m}(t, s)
\end{aligned}
$$

for $n=2$.

This means that the inequality

$$
K_{n-2}(x, s)<\sum_{m=0}^{n-2}\left[\begin{array}{c}
n-2 \\
m
\end{array}\right]_{q} K_{n-m-2}(x, t) K_{m}(t, s)
$$

holds for $n=3$. Our aim is now to use induction, and we assume that (19) holds for $n=l-1$, $l \geq 3$, and we will prove that it then holds also for $n=l$.

We use our induction assumption, make some calculations and obvious estimates and find that

$$
\begin{aligned}
K_{l-1}(x, s)= & K_{l-2}(x, s)\left(x-q^{l-1} s\right) \\
< & \left(\sum_{m=0}^{l-2}\left[\begin{array}{c}
l-2 \\
m
\end{array}\right]_{q} K_{l-m-2}(x, t) K_{m}(t, s)\right)\left(x-q^{l-1} s\right) \\
< & \sum_{m=0}^{l-2}\left[\begin{array}{c}
l-2 \\
m
\end{array}\right]_{q} K_{l-m-2}(x, t) K_{m}(t, s)\left(x-q^{l-m-1} t+q^{l-m-2} t-q^{l-1} s\right) \\
= & \sum_{m=0}^{l-2}\left[\begin{array}{c}
l-2 \\
m
\end{array}\right]_{q} K_{l-m-2}(x, t) K_{m}(t, s)\left(x-q^{l-m-1} t\right) \\
& +\sum_{m=0}^{l-2}\left[\begin{array}{c}
l-2 \\
m
\end{array}\right]_{q} K_{l-m-2}(x, t) K_{m}(t, s) q^{l-m-2}\left(t-q^{m+1} s\right)
\end{aligned}
$$




$$
\begin{aligned}
= & {\left[\begin{array}{c}
l-2 \\
0
\end{array}\right]_{q} K_{l-1}(x, t) K_{0}(t, s)+\sum_{m=1}^{l-2}\left[\begin{array}{c}
l-2 \\
m
\end{array}\right]_{q} K_{l-m-1}(x, t) K_{m}(t, s) } \\
& +\sum_{m=1}^{l-2} q^{l-m-2}\left[\begin{array}{c}
l-2 \\
m-1
\end{array}\right]_{q} K_{l-m-1}(x, t) K_{m}(t, s)+\left[\begin{array}{l}
l-2 \\
l-2
\end{array}\right]_{q} K_{0}(x, t) K_{l-1}(t, s) \\
= & {\left[\begin{array}{c}
l-1 \\
0
\end{array}\right]_{q} K_{l-1}(x, t) K_{0}(t, s) } \\
& +\sum_{m=1}^{l-2}\left(q^{l-m-2}\left[\begin{array}{c}
l-2 \\
m-1
\end{array}\right]_{q}+\left[\begin{array}{c}
l-2 \\
m
\end{array}\right]_{q}\right) K_{l-m-1}(x, t) K_{m}(t, s) \\
& +\left[\begin{array}{c}
l-1 \\
l-1
\end{array}\right]_{q} K_{0}(x, t) K_{l}(t, s) .
\end{aligned}
$$

Since, for any $m \geq 1\left(q^{l-m-2}\left[\begin{array}{c}l-2 \\ m-1\end{array}\right]_{q}+\left[\begin{array}{c}l-2 \\ m\end{array}\right]_{q}=\left[\begin{array}{c}l-1 \\ m\end{array}\right]_{q}\right)$, we get that

$$
K_{l-1}(x, s)<\sum_{m=0}^{l-1}\left[\begin{array}{c}
l-1 \\
m
\end{array}\right]_{q} K_{l-m-1}(x, t) K_{m}(t, s) .
$$

Hence, (19) holds also with $n=l$ which, by the induction axiom, means that also the upper estimate in (15) is proved. The proof is complete.

Proof of Lemma 3.4 From (6) and (7) it follows that

$$
I(z)=(1-q)^{\alpha+\beta}\left(\sum_{q^{j} \leq z} q^{j} f\left(q^{j}\right)\right)^{\alpha}\left(\sum_{q^{i} \geq z} q^{i} g\left(q^{i}\right)\right)^{\beta} .
$$

If $z=q^{k}$, then, for $k \in \mathbb{Z}$,

$$
I(z)=(1-q)^{\alpha+\beta}\left(\sum_{j=k}^{\infty} q^{j} f\left(q^{j}\right)\right)^{\alpha}\left(\sum_{i=-\infty}^{k} q^{i} g\left(q^{i}\right)\right)^{\beta} .
$$

If $q^{k}<z<q^{k-1}$, then, for $k \in \mathbb{Z}$,

$$
I(z)=(1-q)^{\alpha+\beta}\left(\sum_{j=k}^{\infty} q^{j} f\left(q^{j}\right)\right)^{\alpha}\left(\sum_{i=-\infty}^{k-1} q^{i} g\left(q^{i}\right)\right)^{\beta} .
$$

Hence, for $k \in \mathbb{Z}$ and $\beta>0$, we find that

$$
\sup _{q^{k} \leq z<q^{k-1}} I(z)=(1-q)^{\alpha+\beta}\left(\sum_{j=k}^{\infty} q^{j} f\left(q^{j}\right)\right)^{\alpha}\left(\sum_{i=-\infty}^{k} q^{i} g\left(q^{i}\right)\right)^{\beta} .
$$

Therefore

$$
\begin{aligned}
\sup _{z>0} I(z) & =\sup _{k \in \mathbb{Z}} \sup _{q^{k} \leq z<q^{k-1}} I(z) \\
& =(1-q)^{\alpha+\beta} \sup _{k \in \mathbb{Z}}\left(\sum_{j=k}^{\infty} q^{j} f\left(q^{j}\right)\right)^{\alpha}\left(\sum_{i=-\infty}^{k} q^{i} g\left(q^{i}\right)\right)^{\beta} .
\end{aligned}
$$


We have proved that (16) holds wherever $\beta>0$.

Next we assume that $\alpha>0$. Let $q^{k+1}<z<q^{k}, k \in \mathbb{Z}$. Then we get that

$$
I(z)=(1-q)^{\alpha+\beta} \sup _{k \in \mathbb{Z}}\left(\sum_{j=k+1}^{\infty} q^{j} f\left(q^{j}\right)\right)^{\alpha}\left(\sum_{i=-\infty}^{k} q^{i} g\left(q^{i}\right)\right)^{\beta}
$$

and analogously as above we find that

$$
\sup _{q^{k+1}<z \leq q^{k}} I(z)=(1-q)^{\alpha+\beta}\left(\sum_{j=k}^{\infty} q^{j} f\left(q^{j}\right)\right)^{\alpha}\left(\sum_{i=-\infty}^{k} q^{i} g\left(q^{i}\right)\right)^{\beta},
$$

and (16) holds also for the case $\alpha>0$. The proof is complete.

Proof of Lemma 3.5 Let $z=q^{k}, k \in \mathbb{Z}$. By using (6) and (7) we have that

$$
\mathcal{I}^{+}(z)=\left((1-q) \sum_{j=-\infty}^{k} q^{j} g\left(q^{j}\right)\left((1-q) \sum_{i=k}^{\infty} q^{i} K\left(q^{j}, q^{i}\right) f\left(q^{i}\right)\right)^{\alpha}\right)^{\beta} .
$$

For the cases $q^{k+1}<z<q^{k}, k \in Z$ and $q^{k}<z<q^{k-1}, k \in Z$, we find that

$$
\mathcal{I}^{+}(z)=\left((1-q) \sum_{j=-\infty}^{k} q^{j} g\left(q^{j}\right)\left((1-q) \sum_{i=k+1}^{\infty} q^{i} K\left(q^{j}, q^{i}\right) f\left(q^{i}\right)\right)^{\alpha}\right)^{\beta}
$$

and

$$
\mathcal{I}^{+}(z)=\left((1-q) \sum_{j=-\infty}^{k-1} q^{j} g\left(q^{j}\right)\left((1-q) \sum_{i=k}^{\infty} q^{i} K\left(q^{j}, q^{i}\right) f\left(q^{i}\right)\right)^{\alpha}\right)^{\beta},
$$

respectively.

Hence, we conclude that

$$
\sup _{q^{k+1}<z<q^{k-1}} \mathcal{I}^{+}(z)=\left((1-q) \sum_{j=-\infty}^{k} q^{j} g\left(q^{j}\right)\left((1-q) \sum_{i=k}^{\infty} q^{i} K\left(q^{j}, q^{i}\right) f\left(q^{i}\right)\right)^{\alpha}\right)^{\beta} .
$$

Since $\sup _{z>0} \mathcal{I}^{+}(z)=\sup _{k \in Z} \sup _{q^{k+1}<z<q^{k-1}} \mathcal{I}^{+}(z)$, we find that (17) holds. The identity (18) can be proved in a similar way as (17). The proof is complete.

Proof of Lemma 3.6 Without loss of generality we may assume that $Q_{m}^{n-1}<\infty$. By using (5), (6) and (7) we can deduce that

$$
\begin{aligned}
Q_{m}^{n-1}= & \left\{\sum_{i=-\infty}^{\infty}\left(\int_{0}^{\infty} \mathcal{X}_{\left(0, q^{i}\right]}(s) K_{m}^{p^{\prime}}\left(q^{i}, s\right) v^{-p^{\prime}}(s) d_{q} s\right)^{\frac{p(r-1)}{p-r}}\right. \\
& \times\left(\int_{0}^{\infty} \mathcal{X}_{\left[q^{i}, \infty\right)}(x) K_{n-m-1}^{r}\left(x, q^{i}\right) u^{r}(x) d_{q} x\right)^{\frac{p}{p-r}} \\
& \times\left(\int_{0}^{\infty} \mathcal{X}_{\left(0, q^{i}\right]}(s) K_{m}^{p^{\prime}}\left(q^{i}, s\right) v^{-p^{\prime}}(s) d_{q} s\right.
\end{aligned}
$$




$$
\begin{aligned}
& \left.\left.-\int_{0}^{\infty} \mathcal{X}_{\left(0, q^{i+1}\right]}(s) K_{m}^{p^{\prime}}\left(q^{i+1}, s\right) v^{-p^{\prime}}(s) d_{q} s\right)\right\}^{\frac{p-r}{p r}} \\
= & \left\{\sum_{i=-\infty}^{\infty}\left((1-q) \sum_{t=i}^{\infty} q^{t} K_{m}^{p^{\prime}}\left(q^{i}, q^{t}\right) v^{-p^{\prime}}\left(q^{t}\right)\right)^{\frac{p(r-1)}{p-r}}\right. \\
& \times\left((1-q) \sum_{j=-\infty}^{i} q^{j} K_{n-m-1}^{r}\left(q^{j}, q^{i}\right) u^{r}\left(q^{j}\right)\right)^{\frac{p}{p-r}} \\
& \left.\times \Delta^{+}\left(\sum_{n=i}^{\infty}(1-q) q^{n} K_{m}^{r}\left(q^{i}, q^{n}\right) v^{-p^{\prime}}\left(q^{n}\right)\right)\right\}^{\frac{p-r}{p r}},
\end{aligned}
$$

and the first equality in Lemma 3.6 is proved.

The second inequality can be proved in a similar way, so we leave out the details. The proof is complete.

Proof of Theorem 3.1 By using formulas (4) and (6) we find that inequality (13) can be rewritten as

$$
\begin{aligned}
& \left(\sum_{j=-\infty}^{\infty}(1-q)^{r+1} q^{j} u^{r}\left(q^{j}\right)\left(\sum_{i=j}^{\infty} q^{i} f\left(q^{i}\right) K_{n-1}\left(q^{j}, q^{i}\right)\right)^{r}\right)^{\frac{1}{r}} \\
& \quad \leq C\left(\sum_{i=-\infty}^{\infty}(1-q) q^{i} f^{p}\left(q^{i}\right) v^{p}\left(q^{i}\right)\right)^{\frac{1}{p}} .
\end{aligned}
$$

Let

$$
\begin{aligned}
& u_{j}^{r}=(1-q)^{r+1} q^{j} u^{r}\left(q^{j}\right), \quad f_{i}=q^{i} f\left(q^{i}\right), \\
& v_{i}^{p}=(1-q) q^{i(1-p)} v^{p}\left(q^{i}\right), \quad W^{(n)}(i, j)=K_{n-1}\left(q^{j}, q^{i}\right) .
\end{aligned}
$$

Then we get that inequality (21) can be rewritten as the discrete weighted Hardy-type inequality (see, e.g., [26])

$$
\left(\sum_{j=-\infty}^{\infty} u_{j}^{r}\left(\sum_{i=j}^{\infty} W^{(n)}(i, j) f_{i}\right)^{r}\right)^{\frac{1}{r}} \leq C\left(\sum_{i=-\infty}^{\infty} v_{i}^{p} a_{i}^{p}\right)^{\frac{1}{p}} .
$$

Hence, inequality (13) is equivalent to inequality $(23)$, where $\left(W^{(n)}(i, j)\right)$ is the nonnegative triangular matrix which has entries $W^{(n)}(i, j) \geq 0$ for $j \leq i$ and $W^{(n)}(i, j) \equiv 0$ for $j>i$ and is non-decreasing in the first index for all $i \geq j>-\infty$.

First we will prove that, for $n \in N$,

$$
(1-q)^{n-1} \sum_{k_{n-1}=j}^{i}[n-1]_{q} q^{k_{n-1}} \sum_{k_{n-2}=k_{n-1}}^{i}[n-2]_{q} q^{k_{n-2}} \cdots \sum_{k_{1}=k_{2}}^{i}[1]_{q} q^{k_{1}}=W^{(n)}(i, j) .
$$

We will use induction and first we note that $W^{(0)}(i, j)=\left(q^{j}-q^{i}\right)_{q}^{0} \equiv 1$ for $n=1$. If $n=2$, then

$$
(1-q) \sum_{k_{1}=j}^{i} q^{k_{1}}=\sum_{k_{1}=j}^{i}[1]_{q}\left(q^{k_{1}}-q^{k_{1}+1}\right)=\left(q^{j}-q^{i+1}\right)_{q}^{1}=W^{(1)}(i, j) .
$$


Assume now that formula (24) holds for $n-1 \in N$, i.e., that

$$
(1-q)^{n-2} \sum_{k_{n-2}=j}^{i}[n-2]_{q} q^{k_{n-2}} \sum_{k_{n-3}=k_{n-2}}^{i}[n-3]_{q} q^{k_{n-3}} \cdots \sum_{k_{1}=k_{2}}^{i}[1]_{q} q^{k_{1}}=W^{(n-1)}(i, j) .
$$

By using this induction assumption we find that

$$
\begin{aligned}
& (1-q)^{n-1} \sum_{k_{n-1}=j}^{i}[n-1]_{q} q^{k_{n-1}} \sum_{k_{n-2}=k_{n-1}}^{i}[n-2]_{q} q^{k_{n-2}} \ldots \sum_{k_{1}=k_{2}}^{i}[1]_{q} q^{k_{1}} \\
& =(1-q)[n-1]_{q} \sum_{k_{n-1}=j}^{i} q^{k_{n-1}} W^{(n-1)}(i, j) \\
& =\left(1-q^{n-1}\right) \sum_{k_{n-1}=j}^{i} q^{k_{n-1}}\left(q^{k_{n-1}}-q^{i+1}\right)_{q}^{n-2} .
\end{aligned}
$$

Since $\left(q^{k_{n-1}}-q^{i+1}\right)_{q}^{n-1}-\left(q^{k_{n-1}+1}-q^{i+1}\right)_{q}^{n-1}=\left(1-q^{n-1}\right) q^{k_{n-1}}\left(q^{k_{n-1}}-q^{i+1}\right)_{q}^{n-2}$, we get that (24) holds also for $n$. Hence, by the induction axiom, we conclude that (24) holds for each $n \in N$.

Let $w_{m, k}=[m]_{q}\left(q^{k_{m}}-q^{k_{m}+1}\right), m=1,2,3, \ldots, n-1$. Then, by using (24), we have that

$$
W^{(n)}(i, j)=\sum_{k_{n-1}=j}^{i} w_{n-1, k_{n-1}} \sum_{k_{n-2}=k_{n-1}}^{i} w_{n-2, k_{n-2}} \cdots \sum_{k_{1}=k_{2}}^{i} w_{1, k_{1}}
$$

Therefore, we see that the matrix operator in (23), defined by

$$
(\widetilde{S} f)_{j}:=\sum_{i=j}^{\infty} W^{(n)}(i, j) f_{i}, \quad j \in Z
$$

is an $n$-multiple discrete Hardy operator with weights (see (25)).

Therefore, Lemma 2.1 and Lemma 2.2 can be used.

(i) Let $1<r<p<\infty$. Then, based on Lemma 2.2, it follows that inequality (23) holds if and only if $\widetilde{Q}_{n-1}=\max _{0 \leq m \leq n-1} \widetilde{Q}_{m}^{n-1}<\infty$, where

$$
\begin{aligned}
\widetilde{Q}_{m}^{n-1}= & \left\{\sum_{i=-\infty}^{\infty}\left(\sum_{j=i}^{\infty}\left(W^{(m+1)}(j, i)\right)^{p^{\prime}} v_{j}^{-p^{\prime}}\right)^{\frac{p(r-1)}{p-r}}\right. \\
& \times\left(\sum_{k=-\infty}^{i}\left(W^{(n-m)}(i, k)\right)^{r} u_{k}^{r}\right)^{\frac{p}{p-r}} \\
& \left.\times \Delta^{+}\left(\sum_{j=i}^{\infty}\left(W^{(m+1)}\right)^{p^{\prime}}(j, i) v_{j}^{-p^{\prime}}\right)\right\}^{\frac{p-r}{p r}} .
\end{aligned}
$$

Since inequality (23) is equivalent to inequality (13), we conclude that the condition $\widetilde{Q}_{n-1}<\infty$ is a necessary and sufficient condition for the validity of inequality (13). Moreover, $\widetilde{Q}_{n-1} \approx C$. 
By using the definitions (22) in $\widetilde{Q}_{m}^{n-1}$, we get that

$$
\begin{aligned}
\widetilde{Q}_{m}^{n-1}= & \left\{\sum_{i=-\infty}^{\infty}\left((1-q) \sum_{j=i}^{\infty} q^{j} K_{m}^{p^{\prime}}\left(q^{i}, q^{j}\right) v^{-p^{\prime}}\left(q^{j}\right)\right)^{\frac{p(r-1)}{p-r}}\right. \\
& \times\left((1-q) \sum_{k=-\infty}^{i} q^{k} K_{n-m-1}^{r}\left(q^{k}, q^{i}\right) u^{r}\left(q^{k}\right)\right)^{\frac{p}{p-r}} \\
& \left.\times \Delta^{+}\left(\sum_{j=i}^{\infty}(1-q) q^{j} K_{m}^{p^{\prime}}\left(q^{i}, q^{j}\right) v^{-p^{\prime}}\left(q^{j}\right)\right)\right\}^{\frac{p-r}{p r}} .
\end{aligned}
$$

By using Lemma 3.6, we find that

$$
\begin{aligned}
\widetilde{Q}_{m}^{n-1}= & \left\{\int_{0}^{\infty}\left(\int_{0}^{\infty} \mathcal{X}_{(0 ; z]}(s) K_{m}^{p^{\prime}}(z, s) v^{-p^{\prime}}(s) d_{q} s\right)^{\frac{p(r-1)}{p-r}}\right. \\
& \times\left(\int_{0}^{\infty} \mathcal{X}_{[z, \infty)}(x) K_{n-m-1}^{r}(x, z) u^{r}(x) d_{q} x\right)^{\frac{p}{p-r}} \\
& \left.\times D_{q}\left(\int_{0}^{\infty} \mathcal{X}_{(0 ; z]}(s) K_{m}^{p^{\prime}}(z, s) v^{-p^{\prime}}(s) d_{q} s\right)\right\}^{\frac{p-r}{p r}},
\end{aligned}
$$

i.e., that $\widetilde{Q}_{n-1}=Q_{n-1}$. Then we find that inequality (13) holds if and only if $Q_{n-1}<\infty$. Moreover, $Q_{n-1} \approx C$, where $C$ is the best constant in (13). Thus the proof of the statement (i) of Theorem 3.1 is complete.

(ii) Let $1<p \leq r<\infty$. Then from Lemma 2.1 it follows that inequality (23) holds if and only if $\widetilde{H}_{n-1}=\max _{0 \leq m \leq n-1} \widetilde{H}_{m}^{n-1}<\infty$ holds, where

$$
\begin{aligned}
\widetilde{H}_{m}^{n-1}= & \sup _{k \in \mathbb{Z}}\left(\sum_{i=k}^{\infty}\left(W^{(m+1)}(i, k)\right)^{p^{\prime}} v_{i}^{-p^{\prime}}\right)^{\frac{1}{p^{\prime}}} \\
& \times\left(\sum_{j=-\infty}^{k}\left(W^{(n-m)}(k, j)\right)^{r} u_{j}^{r}\right)^{\frac{1}{r}}, \quad n \in \mathbb{N} .
\end{aligned}
$$

If $x=q^{j}, s=q^{i}, t=q^{k}$, for $j \leq k \leq i$, then, by Lemma 3.3 and (22), we obtain that (recall that $\left.W^{(n)}(i, j)=K_{n-1}\left(q^{j}, q^{i}\right)\right)$

$$
W^{(n)}(i, j) \leq \sum_{m=0}^{n-1}\left[\begin{array}{c}
n-1 \\
m
\end{array}\right]_{q} W^{(n-m)}(k, j) W^{(m+1)}(i, k) .
$$

Since, again by Lemma 3.3, $\max _{0 \leq m \leq n-1} W^{(m+1)}(i, k) W^{(n-m)}(k, j) \leq W^{(n)}(i, j)$, it follows that

$$
W^{(n)}(i, j) \geq h(n) \sum_{m=0}^{n-1}\left[\begin{array}{c}
n-1 \\
m
\end{array}\right]_{q} W^{(m+1)}(i, k) W^{(n-m)}(k, j),
$$

where $h(n)=\left(\sum_{m=0}^{n-1}\left[\begin{array}{c}n-1 \\ m\end{array}\right]_{q}\right)^{-1}$. 
According to (26) and (27) we have that

$$
W^{(n)}(i, j) \approx \sum_{m=0}^{n-1} W^{(m)}(i, k) \mathcal{W}^{(n, m)}(k, j)
$$

where $\mathcal{W}^{(n, m)}(k, j)=\left[\begin{array}{c}n-1 \\ m\end{array}\right]_{q} W^{(n-m-1)}(k, j)$.

Therefore, we have proved that the matrix $\left(W^{(n)}(i, j)\right)$ in $(23)$ satisfies the Oinarov condition (11) and Lemma 2.4 can be used.

Hence, we have the following necessary and sufficient conditions for the validity of inequality (23):

$$
\widetilde{A}^{+}=\sup _{k \in Z}\left(\sum_{j=-\infty}^{k} u_{j}^{r}\left(\sum_{i=k}^{\infty}\left(W^{(n)}(i, j)\right)^{p^{\prime}} v_{i}^{-p^{\prime}}\right)^{\frac{r}{p^{\prime}}}\right)^{\frac{1}{r}}<\infty
$$

or

$$
\tilde{A}^{-}=\sup _{k \in Z}\left(\sum_{i=k}^{\infty} v_{i}^{-p^{\prime}}\left(\sum_{j=-\infty}^{k}\left(W^{(n)}(i, j)\right)^{r} u_{j}^{r}\right)^{\frac{p^{\prime}}{r}}\right)^{\frac{1}{p^{\prime}}}<\infty .
$$

Since, again by Lemma 3.3, $\max _{0 \leq m \leq n-1} W^{(m+1)}(i, k) W^{(n-m)}(k, j) \leq W^{(n)}(i, j)$, we get that

$$
\begin{aligned}
\widetilde{H}_{n-1} & =\sup _{k \in Z}\left(\sum_{j=-\infty}^{k} u_{j}\left(\sum_{i=k}^{\infty}\left(\max _{0 \leq m \leq n-1}\left(W^{(n-m)}(k, j) W^{(m+1)}(i, k)\right)\right)^{p^{\prime}} v_{i}^{-p^{\prime}}\right)^{\frac{r}{p^{\prime}}}\right)^{\frac{1}{r}} \\
& \leq\left(\sum_{j=-\infty}^{k} u_{j}\left(\sum_{i=k}^{\infty}\left(W^{(n)}(i, j)\right)^{p^{\prime}} v_{i}^{-p^{\prime}}\right)^{\frac{r}{p^{\prime}}}\right)^{\frac{1}{r}}=\widetilde{A}^{+} .
\end{aligned}
$$

Moreover, by (26) we have that

$$
\begin{aligned}
\widetilde{A}^{+} & \leq \sup _{k \in Z}\left(\sum_{j=-\infty}^{k} u_{j}\left(\sum_{i=k}^{\infty}\left(\sum_{m=0}^{n-1}\left[\begin{array}{c}
n-1 \\
m
\end{array}\right]_{q} W^{(n-m)}(k, j) W^{(m+1)}(i, k)\right)^{p^{\prime}} v_{i}^{-p^{\prime}}\right)^{\frac{r}{p^{\prime}}}\right)^{\frac{1}{r}} \\
& \leq \sum_{m=0}^{n-1}\left[\begin{array}{c}
n-1 \\
m
\end{array}\right]_{q} \widetilde{H}_{n-1} \ll \widetilde{H}_{n-1} .
\end{aligned}
$$

Hence, $\widetilde{A}^{+} \approx \widetilde{H}_{n-1}$. In a similar way it can be proved that $\widetilde{H}_{n-1} \approx \widetilde{A}^{-}$.

Since inequality (13) is equivalent to inequality (23), we get that inequality (13) holds if and only if at least one of the conditions $\widetilde{A}^{+}<\infty$ or $\widetilde{A}^{-}<\infty$, or $\widetilde{H}_{n-1}<\infty$ holds.

Now, using notations (22) in $\widetilde{H}_{m}^{n-1}$, we obtain that

$$
\begin{aligned}
\widetilde{H}_{m}^{n-1}= & (1-q)^{\frac{1}{r}+\frac{1}{p^{\prime}}} \sup _{k \in \mathbb{Z}}\left(\sum_{j=-\infty}^{k} q^{j} u^{r}\left(q^{j}\right) K_{n-m-1}^{r}\left(q^{j}, q^{k}\right)\right)^{\frac{1}{r}} \\
& \times\left(\sum_{i=k}^{\infty} q^{t} v^{-p^{\prime}}\left(q^{i}\right) K_{m}^{p^{\prime}}\left(q^{k}, q^{i}\right)\right)^{\frac{1}{p^{\prime}}} .
\end{aligned}
$$


The parameters and functions in $\widetilde{H}_{m}^{n-1}$ satisfy all the conditions of Lemma 3.4. Therefore, we find that

$$
\begin{aligned}
\widetilde{H}_{m}^{n-1}= & \sup _{z>0}\left(\int_{0}^{\infty} \mathcal{X}_{[z, \infty)}(x) K_{n-m-1}^{r}(x, z) u^{r}(x) d_{q} x\right)^{\frac{1}{r}} \\
& \times\left(\int_{0}^{\infty} \mathcal{X}_{(0, z]}(s) K_{m}^{p^{\prime}}(z, s) v^{-p^{\prime}}(s) d_{q} s\right)^{\frac{1}{p^{\prime}}}
\end{aligned}
$$

i.e., that $\widetilde{H}_{m}^{n-1}=H_{m}^{n-1}$ and $\widetilde{H}_{n-1}=H_{n-1}=\max _{0 \leq m \leq n-1} H_{m}^{n-1}<\infty$.

In a similar way as above, by using Lemma 3.5 and (22), we get that $A_{q}^{+}=\widetilde{A}^{+}$and $A_{q}^{-}=\widetilde{A}^{-}$.

Hence, we obtain that inequality (13) holds if and only if at least one of the conditions $H_{n-1}<\infty$ or $A_{q}^{+}<\infty$, or $A_{q}^{-}<\infty$ holds. Moreover, $A_{q}^{+} \approx A_{q}^{-} \approx H_{n-1} \approx C$, where $C$ is the best constant in (13). Also the proof of the statement (ii) of Theorem 3.1 is complete.

Proof of Theorem 3.2 In a similar way as in the proof of Theorem 3.1, by using (4), (7) and (22), we can prove that we have the following discrete Hardy-type inequality:

$$
\left(\sum_{i=-\infty}^{\infty} u_{i}^{r}\left(\sum_{j=-\infty}^{i} W_{n-1,1}^{(n)}(i, j) f_{i}\right)^{r}\right)^{\frac{1}{r}} \leq C\left(\sum_{i=-\infty}^{\infty} v_{i}^{p} a_{i}^{p}\right)^{\frac{1}{p}},
$$

which is equivalent to inequality (14).

(i) Let $1<r<p<\infty$. By using Lemma 2.2 and Lemma 3.6, we can in a similar way as in the proof of Theorem 3.1(i) derive that inequality (14) holds if and only if $\mathcal{Q}_{n-1}<\infty$ holds. Moreover, $\mathcal{Q}_{n-1} \approx C^{*}$, where $C^{*}$ is the best constant in (14). The proof of part (i) is complete.

(ii) Let $1<p \leq r<\infty$. By using Lemma 2.4, Lemma 2.1, Lemma 3.4 and Lemma 3.5, we can, analogously as in the proof of the (ii)-part, prove that inequality (14) holds if and only if at least one of the conditions $\mathcal{H}_{n-1}<\infty$ or $\mathcal{A}_{q}^{+}<\infty$, or $\mathcal{A}_{q}^{-}<\infty$ holds. Moreover, $\mathcal{H}_{n-1} \approx \mathcal{A}_{q}^{-} \approx \mathcal{A}_{q}^{+} \approx C^{*}$, where $C^{*}$ is the best constant in (14). The proof of part (ii) is complete.

\section{Competing interests}

The authors declare that they have no competing interests.

\section{Authors' contributions}

All authors have on equal level discussed and posed the research questions in this paper. SS is the main author concerning the proofs of the main results and typing of the manuscript. L-EP has put the results into a more general frame in the introduction and instructed how to write the paper in this final form. All authors read and approved the final manuscript.

\section{Author details}

${ }^{1}$ Luleå University of Technology, Luleå, 971 87, Sweden. ${ }^{2}$ Narvik University College, P.O. Box 385, Narvik, 8505, Norway.

${ }^{3}$ L.N. Gumilyov Eurasian National University, Munaytpasov St. 5, Astana, 010008, Kazakhstan.

\section{Acknowledgements}

We thank both careful referees and Professor Ryskul Oinarov for generous advice, which have improved the final version of this paper.

Received: 6 May 2015 Accepted: 7 September 2015 Published online: 24 September 2015

\section{References}

1. Jackson, FH: On q-definite integrals. Q. J. Pure Appl. Math. 41, 193-203 (1910)

2. Cheung, P, Kac, V: Quantum Calculus. Edwards, Ann Arbor (2000) 
3. Annaby, MH, Mansour, ZS: q-Fractional Calculus and Equations. Springer, Heidelberg (2012)

4. Bangerezako, G: Variational calculus on q-nonuniform lattices. J. Math. Anal. Appl. 306, 161-179 (2005)

5. Ernst, T: A Comprehensive Treatment of $q$-Calculus. Birkhäuser, Basel (2012)

6. Ernst, T: The history of $q$-calculus and a new method. PhD thesis, Uppsala University (2001)

7. Exton, H: q-Hypergeometric Functions and Applications. Halsted, New York (1983)

8. Gauchman, H: Integral inequalities in q-calculus. Comput. Math. Appl. 47, 281-300 (2004)

9. Krasniqi, V, Erratum: Several q-integral inequalities. J. Math. Inequal. 5(3), 451 (2011)

10. Miao, Y, Qi, F: Several q-integral inequalities. J. Math. Inequal. 3, 115-121 (2009)

11. Stanković, MS, Rajković, PM, Marinković, SD: On q-fractional derivatives of Riemann-Liouville and Caputo type (2013). arXiv:0909.0387

12. Sulaiman, WT: New types of $q$-integral inequalities. Adv. Pure Math. 1, 77-80 (2011)

13. Maligranda, L, Oinarov, R, Persson, L-E: On Hardy q-inequalities. Czechoslov. Math. J. 64, 659-682 (2014)

14. Hardy, GH: Note on a theorem of Hilbert. Math. Z. 6, 314-317 (1920)

15. Hardy, GH: Notes on some points in the integral calculus, LX. An inequality between integrals. Messenger Math. 54, 150-156 (1925)

16. Kokilashvili, V, Meskhi, A, Persson, L-E: Weighted Norm Inequalities for Integral Transforms with Product Kernels. Nova Science Publishers, New York (2010)

17. Kufner, A, Maligranda, A, Persson, L-E: The Hardy Inequality - About Its History and Some Related Results. University of West Bohemia, Plzen (2007)

18. Kufner, A, Persson, L-E: Weighted Inequalities of Hardy Type. World Scientific, River Edge (2003)

19. Al-Salam, WA: Some fractional $q$-integrals and q-derivatives. Proc. Edinb. Math. Soc. 15, 135-140 (1966/1967)

20. Oinarov, R, Temirkhanova, AM: Boundedness and compactness of a class of matrix operators in weighted sequence spaces. J. Math. Inequal. 2, 555-570 (2008)

21. Kalybay, A, Oinarov, R, Temirkhanova, A: Boundedness of $n$-multiple discrete Hardy operators with weights for $1<q<p<\infty$. J. Funct. Spaces Appl. 41, 1-9 (2013)

22. Oinarov, R, Taspaganbetova, Z: Criteria of boundedness and compactness of a class of matrix operators. J. Inequal. Appl. 2012, 53 (2012)

23. Stepanov, VD: Two-weighted estimates of Riemann-Liouville integrals. Math. USSR, Izv. 36, 669-681 (1991)

24. Stepanov, VD: Weighted inequalities for a class of Volterra convolution operators. J. Lond. Math. Soc. 45, 232-242 (1992)

25. Baiaristanov, AO, Shaimardan, S, Temirkhanova, A: Weighted Hardy inequalities in quantum analysis. Vestin. KarGU, Math. Ser. 70, 35-45 (2013)

26. Persson, L-E, Ragusa, MA, Samko, N, Wall, P: Commutators of Hardy operators in vanishing Morrey spaces. In: 9th International Conference on Mathematical Problems in Engineering, Aerospace and Sciences (ICNPAA 2012). AIP Conference Proceedings, vol. 1493, pp. 859-866 (2012)

\section{Submit your manuscript to a SpringerOpen ${ }^{\circ}$ journal and benefit from:}

- Convenient online submission

Rigorous peer review

- Immediate publication on acceptance

- Open access: articles freely available online

- High visibility within the field

- Retaining the copyright to your article 